\title{
NUMERICAL SIMULATION OF A VISCOELASTIC FLUID WITH A PRECONDITIONED SCHWARZ METHOD
}

\author{
LUÍS BORGES \\ Inst. Superior Eng. Lisboa \\ Área de Mat. and CEMAT/IST \\ R. Cons. E. Navarro,1 Lisboa, Portugal \\ E-mail:lborges@math.ist.utl.pt \\ ADÉLIA SEQUEIRA \\ Dept. Matemática and CEMAT \\ Inst. Superior Técnico/UTL \\ Av. Rovisco Pais, 1049-001 Lisboa, Portugal \\ E-mail: adelia.sequeira@math.ist.utl.pt
}

\begin{abstract}
In this paper we apply a domain decomposition method to approach the solution of a non-Newtonian viscoelastic Oldroyd-B model. The numerical scheme is based on a fixed-point argument applied to the original non-linear system of partial differential equations decoupled into a Navier-Stokes system and a tensorial transport equation. Using a modified Schwarz algorithm, involving block preconditioners for the Navier-Stokes equations, the decoupled problems are solved iteratively. Numerical simulations on a 4:1 abrupt contraction flow problem are considered to validate the scheme.
\end{abstract}

1. Introduction. Domain Decomposition Methods (DDM) applied to the numerical solution of large-scale algebraic systems arising from the approximation of partial differential equations have been intensively studied by many authors (see e.g [18], [8], [23] and the references cited therein). They are based on a decomposition of the spatial domain of the problem into several subdomains, which may or may not overlap, and consist in solving reduced size subproblems on these subdomains, while enforcing suitable continuity

2000 Mathematics Subject Classification: Primary 46C20; Secondary 32G81.

Key words and phrases: Oldroyd-B model, finite elements, domain decomposition method, block preconditioners.

This work has been partially supported by the Center for Mathematics and its Applications - CEMAT through FCT's funding program and by the project PTDC/MAT/68166/2006.

The paper is in final form and no version of it will be published elsewhere. 
requirements at the corresponding interfaces. Such reformulations are usually motivated by the construction of new solvers for parallel computing. A multi-domain approach can also account for the solution of heterogeneous models related to physical problems defined in complex geometries and usually leads to the construction of optimal (mesh size independent) preconditioners.

The goal of this paper is to apply a preconditioned DDM to the numerical solution of an incompressible non-Newtonian Oldroyd-B fluid. The investigation of mathematical and numerical methods for the solution of non-Newtonian fluid models is a very rich field of research with many interesting industrial and biological applications. Usually, the constitutive equations lead to highly non-linear systems of partial differential equations of a combined elliptic-hyperbolic type (or parabolic-hyperbolic, for unsteady flows) closed with appropriate boundary (or initial and boundary) conditions.

The hyperbolic nature of the constitutive equations is responsible for many of the difficulties associated with the numerical simulation of viscoelastic flows. Some factors including singularities in the geometry, boundary layers in the flow and the dominance of the non-linear terms in the equations, result in numerical instabilities for high values of the Weissenberg number (nondimensional number related with the elasticity of the fluid). Space discretizations using finite elements, finite volumes or spectral methods together with finite difference or fractional-step schemes in time, for unsteady flows, lead to the solution of algebraic systems, typically very large, that are solved using direct or iterative methods. The solution of these algebraic systems often requires the use of preconditioners that can be regarded as operator projections, multigrid or domain decomposition methods. The major difficulties in many numerical schemes are related to the large amount of computation involved and to the loss of convergence or stability. This is the object of active research in the field (see e.g. [17], [19] and cited references).

In this paper we present a domain decomposition method applied to the numerical approximation of the equations of motion of an incompressible Oldroyd-B fluid model, decoupled into a Navier-Stokes system and a transport equation. Appropriate preconditioned DDM using a modified Schwarz algorithm are introduced for the two auxiliary problems. Numerical results are given in a 4:1 abrupt planar contraction, considered as a good benchmark to validate numerical schemes for viscoelastic flows. Other validation results can be found in [2] and [4].

2. Model formulation. We consider steady isothermal flows of an incompressible Oldroyd-B fluid in a bounded domain $\Omega \subset \mathbb{R}^{2}$ with a polygonal boundary $\partial \Omega$. For these fluids, the extra-stress tensor is related to the kinematic variables through

$$
\mathbf{S}+\lambda_{1} \stackrel{\nabla}{\mathbf{S}}=2 \mu\left(\lambda_{2} \stackrel{\nabla}{D} \mathbf{u}+D \mathbf{u}\right),
$$

where $\mathbf{u}$ is the velocity field, $D \mathbf{u}=\frac{1}{2}\left(\nabla \mathbf{u}+\nabla \mathbf{u}^{t}\right)$ denotes the symmetric part of the velocity gradient, $\mu$ is the constant viscosity and $\lambda_{1}>0, \lambda_{2}>0$ are respectively the relaxation and retardation times. The symbol $\nabla$ denotes the objective derivative of Oldroyd type defined by

$$
\stackrel{\nabla}{\mathbf{S}}=\mathbf{u} \cdot \nabla \mathbf{S}-\mathbf{S} \nabla \mathbf{u}-(\nabla \mathbf{u})^{T} \mathbf{S} .
$$


The Cauchy stress tensor is given by $\mathbf{T}=-p \mathbf{I}+\mathbf{S}$, where $p$ represents the pressure and $\mathbf{S}$ is the extra stress tensor. The equations of conservation of momentum and mass hold in the domain $\Omega$,

$$
\rho \mathbf{u} \cdot \nabla \mathbf{u}+\nabla p=\nabla \cdot \mathbf{S}, \quad \nabla \cdot \mathbf{u}=0
$$

where $\rho>0$ is the (constant) density of the fluid. Decomposing the extra-stress tensor $\mathbf{S}$ into the sum of its Newtonian part $\boldsymbol{\tau}_{s}=2 \mu \frac{\lambda_{2}}{\lambda_{1}} D \mathbf{u}$ and its viscoelastic part $\boldsymbol{\tau}$, we rewrite (1)-(2) as

$$
\begin{cases}-\frac{\lambda_{2}}{\lambda_{1}} \mu \Delta \mathbf{u}+\rho \mathbf{u} \cdot \nabla \mathbf{u}+\nabla p=\nabla \cdot \boldsymbol{\tau} & \text { in } \Omega \\ \nabla \cdot \mathbf{u}=0 & \text { in } \Omega \\ \boldsymbol{\tau}+\lambda_{1} \stackrel{\nabla}{\boldsymbol{\tau}}=2 \mu\left(1-\frac{\lambda_{2}}{\lambda_{1}}\right) D \mathbf{u} & \text { in } \Omega\end{cases}
$$

We consider the nondimensional form of this system by introducing the following quantities

$$
x=\frac{\widetilde{x}}{L}, u=\frac{\widetilde{u}}{U}, p=\frac{\widetilde{p} L}{\mu U}, \boldsymbol{\tau}=\frac{\widetilde{\tau} L}{\mu U},
$$

where the symbol $\sim$ is attached to dimensional parameters ( $L$ represents a reference length and $U$ a characteristic velocity of the flow). We also set

$$
\varepsilon=1-\frac{\lambda_{2}}{\lambda_{1}}
$$

and introduce the Reynolds number and the Weissenberg number

$$
\mathcal{R} e=\frac{\rho U L}{\mu}, \quad \mathcal{W} e=\frac{\lambda_{1} U}{L} .
$$

The nondimensional system takes the form

$$
\begin{cases}-(1-\varepsilon) \Delta \mathbf{u}+\mathcal{R} e \mathbf{u} \cdot \nabla \mathbf{u}+\nabla p=\nabla \cdot \boldsymbol{\tau} & \text { in } \Omega \\ \nabla \cdot \mathbf{u}=0 & \text { in } \Omega \\ \boldsymbol{\tau}+\mathcal{W} e \stackrel{\nabla}{\tau}=2 \varepsilon D \mathbf{u} & \text { in } \Omega\end{cases}
$$

and is composed of a Navier-Stokes system for $(\mathbf{u}, p)$ coupled with a transport equation for $\boldsymbol{\tau}$. This system is supplemented with a Dirichlet boundary condition

$$
\mathbf{u}=\mathbf{g} \text { with } \mathbf{g} \cdot \mathbf{n}=0 \quad \text { on } \partial \Omega,
$$

$\mathbf{n}$ being the unit outward normal to the boundary $\partial \Omega$.

3. Numerical approximation. We consider an approximation of the problem (3) by using finite element methods. Let $\mathcal{T}_{h}$ be a triangulation of $\bar{\Omega}$ with triangles $K$ such that

$$
\bar{\Omega} \approx \overline{\Omega_{h}}=\bigcup_{K \in \mathcal{T}} K
$$

We define the finite element spaces $\mathbf{V}_{h}, M_{h}$ and $\mathcal{E}_{h}$ with dimensions $N^{v}, N^{p}$ and $N^{s}$ to approximate $\mathbf{u}, p$ and $\boldsymbol{\tau}$, respectively, with $h$ being a positive parameter related with the 
size of the mesh. For the approximation of the velocity and pressure fields we consider the so-called Hood-Taylor elements, where the corresponding spaces satisfy the discrete inf-sup condition (see [7]). Recalling the definition of these spaces:

$$
\begin{gathered}
\mathbf{V}_{h}=\left\{\mathbf{v}_{h} \in \mathbf{H}^{1}(\Omega) \cap \mathbf{C}(\bar{\Omega}): \mathbf{v}_{h_{\left.\right|_{K}}} \in \mathbb{P}^{2}, \forall K \in \mathcal{T}_{h}\right\}, \\
M_{h}=\left\{q_{h} \in L_{0}^{2}(\Omega) \cap C(\bar{\Omega}): q_{h_{\left.\right|_{K}}} \in \mathbb{P}^{1}, \forall K \in \mathcal{T}_{h}\right\}
\end{gathered}
$$

and

$$
\mathbf{V}_{h}^{0}=\mathbf{V}_{h} \cap \mathbf{H}_{0}^{1}(\Omega)
$$

For the stress tensor we consider $\mathbb{P}^{1}$ elements continuous in $\bar{\Omega}$. These spaces are defined as follows

$$
\mathcal{E}_{h}=\left\{\boldsymbol{\sigma}_{h} \in \mathcal{E} \cap \mathbf{C}(\bar{\Omega}): \sigma_{h_{\left.\right|_{K}}} \in \mathbb{P}^{1}, \forall K \in \mathcal{T}_{h}\right\}
$$

where

$$
\mathcal{E}=\left\{\boldsymbol{\sigma} \in \mathbf{L}^{2}(\Omega): \mathbf{u} \cdot \nabla \boldsymbol{\sigma} \in \mathbf{L}^{2}(\Omega), \sigma_{12}=\sigma_{21}\right\}
$$

We refer to [21], [3] and [6] for this choice of finite elements.

REMARK 1. For the approximation of the transport equation, an alternative finite element space is defined by using test functions that are in $\mathbf{L}^{2}(\Omega)$, and can be discontinuous across the boundary of the elements (see e.g. [14], [12]).

With the discrete spaces already defined, we consider the following discrete variational problem:

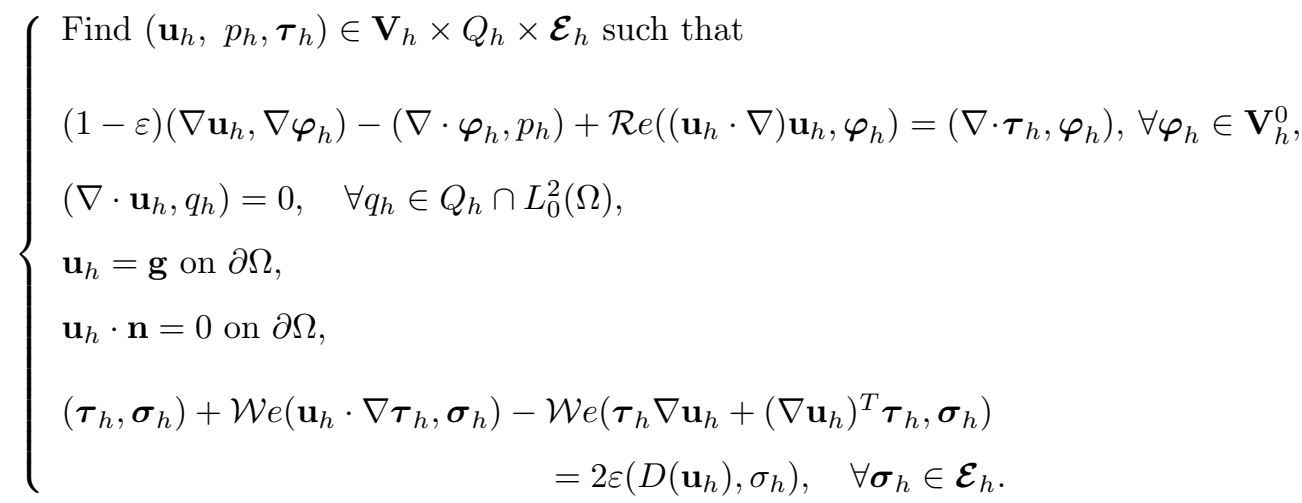

Since this problem is a non-linear system of coupled equations, the numerical scheme to solve (5) is based on a global fixed-point argument used by Najib and Sandri [15] and defined as follows: for a fixed $\boldsymbol{\tau}$, the first two equations (5) define a Navier-Stokes system in the variables $(\mathbf{u}, p)$ and for a fixed $(\mathbf{u}, p)$ the third equation is a transport tensorial equation. This argument, used for the continuous problem is now extended to the discrete problem as follows 
For $k \geqslant 0$

$$
\left\{\begin{array}{l}
\qquad \begin{array}{l}
\text { Fiven } \boldsymbol{\tau}^{k} \text { and } \mathbf{u}^{k}, \\
(1-\varepsilon)\left(\nabla \mathbf{u}_{h}^{k+1}, p^{k+1}\right) \text { solution of } \\
\left(\nabla \cdot \boldsymbol{\varphi}_{h}\right)-\left(\nabla \cdot \boldsymbol{\varphi}_{h}, p_{h}\right)+\mathcal{R} e\left(\left(\mathbf{u}_{h}^{k+1}, \nabla\right) \mathbf{u}_{h}^{k+1}, \boldsymbol{\varphi}_{h}\right)=\left(\nabla \cdot \boldsymbol{\tau}_{h}^{k}, \boldsymbol{\varphi}_{h}\right),
\end{array} \\
\bullet \begin{array}{l}
\text { Given } \boldsymbol{\tau}_{h}^{k} \text { and } \mathbf{u}_{h}^{k+1}, \\
\left(\boldsymbol{\tau}_{h}^{k+1}, \boldsymbol{\sigma}_{h}\right)+\mathcal{W} e\left(\mathbf{u}_{h}^{k+1} \cdot \nabla \boldsymbol{\tau}_{h}^{k+1}, \boldsymbol{\sigma}_{h}\right)=\mathcal{W} e\left(\boldsymbol{\tau}_{h}^{k} \nabla \mathbf{u}_{h}^{k+1}+\left(\nabla \mathbf{u}_{h}^{k+1}\right)^{T} \boldsymbol{\tau}_{h}^{k}, \boldsymbol{\sigma}_{h}\right) \\
+2 \varepsilon\left(D\left(\mathbf{u}_{h}^{k+1}\right), \boldsymbol{\sigma}_{h}\right) .
\end{array}
\end{array}\right.
$$

3.1. Algebraic systems. Taking into account the definition of the finite element spaces $\mathbf{V}_{h}, Q_{h}$ and $\mathcal{E}_{h}$ let us construct the algebraic systems associated to the problem. Let $n$, $m$ and $t$ be the dimensions of finite spaces $\mathbf{V}_{h}, Q_{h}$ and $\mathcal{E}_{h}$, respectively and let

$$
\left\{\boldsymbol{\varphi}_{i}\right\}_{i=1, \ldots, n}, \quad\left\{\psi_{\ell}\right\}_{\ell=1, \ldots, m} \quad \text { and } \quad\left\{\boldsymbol{\sigma}_{i}\right\}_{k=1, \ldots, t}
$$

be their respective bases. Writing the unknowns $(\mathbf{u}, p, \boldsymbol{\tau})$ using the basis functions, we have

$$
\mathbf{u}_{h}=\sum_{i=1}^{n} \mathbf{U}^{i} \boldsymbol{\varphi}_{i}, \quad p_{h}=\sum_{\ell=1}^{m} P^{\ell} \psi_{\ell} \quad \text { and } \boldsymbol{\sigma}_{h}=\sum_{s=1}^{t} T^{j} \boldsymbol{\sigma}_{s} .
$$

Substituting in (5) and setting $\boldsymbol{\sigma}_{h}=\boldsymbol{\sigma}_{i}$ we get a non-linear system

$$
\overline{\mathbf{A}}(\mathbf{u}) X=\overline{\mathbf{H}}
$$

where $X=(\mathbf{U}, P, \mathbf{T})$. Since we decouple the global system using the previous scheme, (8) is decomposed into two algebraic systems, one corresponding to the Navier-Stokes equations

$$
\left(\begin{array}{cc}
\mathbf{D}+\mathbf{C}(\mathbf{U}) & \mathbf{B} \\
\mathbf{B}^{\mathrm{T}} & 0
\end{array}\right)\left(\begin{array}{c}
\mathbf{U} \\
P
\end{array}\right)=\left(\begin{array}{c}
\mathbf{H}(\mathbf{T}) \\
0
\end{array}\right)
$$

and the other to the transport equation

$$
\mathbf{S}(\mathbf{U}) \mathbf{T}=\mathbf{G}(\mathbf{U}, \mathbf{T}) .
$$

The matrices $\mathbf{D}, \mathbf{C}$ and $\mathbf{B}$ refer to the discretizations of the operators $\Delta, \mathbf{u} \cdot \nabla$ and $\nabla$, respectively.

Now the (global) fixed point iteration scheme can be written as follows: 
- For a given initial condition $\left(\mathbf{U}^{0}, \mathbf{T}^{0}\right)$,

$$
\left\{\begin{array}{l}
\left.\bullet \begin{array}{cc}
\bullet & \text { Find } \mathbf{U}^{m+1} \text { solution of } \\
\mathbf{D}+\mathbf{C}\left(\mathbf{U}^{m+1}\right) & \mathbf{B} \\
\mathbf{B}^{\mathrm{T}} & 0
\end{array}\right)\left(\begin{array}{c}
\mathbf{U}^{m+1} \\
P^{m}
\end{array}\right)=\left(\begin{array}{c}
\mathbf{H}\left(\mathbf{T}^{m}\right) \\
0
\end{array}\right) \\
\text { Find } \mathbf{T}^{m+1} \text { solution of } \\
\mathbf{S}\left(\mathbf{U}^{m+1}\right) \mathbf{T}^{m+1}=\mathbf{G}\left(\mathbf{U}^{m+1}, \mathbf{T}^{m}\right) \\
\bullet \text { Do } m=m+1
\end{array}\right.
$$

3.2. Domain Decomposition Method. We consider a non-overlapping decomposition of the domain $\Omega$ into two subdomains $\Omega_{1}$ and $\Omega_{2}$ (both with Lipschitz boundaries), which satisfies the following conditions

$$
\Omega=\Omega_{1} \cup \Omega_{2}, \quad \Omega_{1} \cap \Omega_{2} \neq \emptyset \quad \text { and } \quad \Gamma=\bar{\Omega}_{1} \cap \bar{\Omega}_{2},
$$

where $\Gamma$ denotes the interface.

Rewriting problem (3) in this multi-domain framework, we have the following problem

$$
\begin{cases}\text { Find }\left(\mathbf{u}_{i}, p_{i}, \boldsymbol{\tau}_{i}\right) \in \mathbf{H}^{1}\left(\Omega_{i}\right) \times L_{0}^{2}(\Omega) \times \mathbf{L}^{2}\left(\Omega_{i}\right) \text { such that } & \\ -(1-\varepsilon) \Delta \mathbf{u}_{i}+\nabla p_{i}+\mathcal{R} e\left(\mathbf{u}_{i} \cdot \nabla\right) \mathbf{u}_{i}=\nabla \cdot \boldsymbol{\tau}_{i} & \text { in } \Omega_{i}, \\ \nabla \cdot \mathbf{u}_{i}=0 & \text { in } \Omega_{i}, \\ \mathbf{u}_{i}=\mathbf{g} & \text { on } \partial \Omega_{i} \cap \partial \Omega \\ \mathbf{u}_{i} \cdot \mathbf{n}_{i}=0 & \text { on } \partial \Omega_{i} \cap \partial \Omega \\ \boldsymbol{\tau}_{i}+\mathcal{W} e \mathbf{u}_{i} \cdot \nabla \boldsymbol{\tau}_{i}-\mathcal{W} e\left(\boldsymbol{\tau}_{i} \nabla \mathbf{u}_{i}+\left(\nabla \mathbf{u}_{i}\right)^{T} \boldsymbol{\tau}_{i}\right)=2 \varepsilon D\left(\mathbf{u}_{i}\right) & \text { in } \Omega_{i},\end{cases}
$$

and provide the interface transmission conditions

$$
\begin{cases}\mathbf{u}_{1}=\mathbf{u}_{2} & \text { on } \Gamma, \\ \mathbf{p}_{1}=\mathbf{p}_{2} & \text { on } \Gamma, \\ \mathbf{T}\left(\mathbf{u}_{1}, p_{1}\right) \cdot \mathbf{n}_{1}=\mathbf{T}\left(\mathbf{u}_{2}, p_{2}\right) \cdot \mathbf{n}_{2} & \text { on } \Gamma, \\ \left(\mathbf{u}_{1} \cdot \mathbf{n}_{1}\right) \boldsymbol{\tau}_{1}=\left(\mathbf{u}_{2} \cdot \mathbf{n}_{2}\right) \boldsymbol{\tau}_{2} & \text { on } \Gamma,\end{cases}
$$

where $\mathbf{T}\left(\mathbf{n}_{i}, p_{i}\right) \cdot \mathbf{n}_{i}=\frac{\partial \mathbf{u}_{i}}{\partial n_{i}}-p_{i} \mathbf{n}_{i}, \mathbf{n}_{i}$ being the unit outward normal to $\partial \Omega_{i} \cap \Gamma(i=1,2)$.

3.2.1. Multi-domain approximate problem. The numerical approach of the problem (12) is done by applying DDM to the decoupled auxiliary problems, the Navier-Stokes system (9) and the transport equation (10). For that purpose, let us introduce two triangulations $\mathcal{T}_{1, h}$ and $\mathcal{T}_{2, h}$ of $\Omega_{1}$ and $\Omega_{2}$ with triangles $K$, that satisfy the following assumption: if the subdomains $\Omega_{i}, i=1,2$ have a polygonal boundary, then

$$
\Omega_{i}=\bigcup_{K \in \mathcal{T}_{i, h}} K
$$


otherwise

$$
\Omega_{i} \approx \Omega_{i, h}=\bigcup_{K \in \mathcal{T}_{i, h}} K
$$

where $h=\max _{K \in \mathcal{T}_{i, h}} h_{K}\left(h_{K}\right.$ is diameter of $\left.K\right)$.

For the Navier-Stokes system we define the finite element spaces $\mathbf{V}_{i, h}, Q_{i, h}$ with dimension $N_{i}^{v}, N_{i}^{p}$ to approximate the velocity and pressure, respectively. For the approximation of $u_{i}$ and $p_{i}$ we consider the Hood-Taylor elements, with finite element spaces defined by

$$
\begin{aligned}
\mathbf{V}_{i, h} & =\left\{\mathbf{v}_{i, h} \in \mathbf{H}^{1}\left(\Omega_{i}\right) \cup \mathbf{C}\left(\bar{\Omega}_{i}\right):\left.\mathbf{v}_{i, h}\right|_{K} \in \mathbb{P}^{2}, \forall K \in \mathcal{T}_{i, h}\right\}, \\
Q_{i, h} & =\left\{q_{i, h} \in L^{2}\left(\Omega_{i}\right) \cap C\left(\bar{\Omega}_{i}\right):\left.q_{i, h}\right|_{K} \in \mathbb{P}^{1}, \forall K \in \mathcal{T}_{i, h}\right\} ;
\end{aligned}
$$

we also consider the subspace

$$
\mathbf{V}_{i, h}^{0}=\mathbf{V}_{i, h} \cap \mathbf{H}_{0}^{1}\left(\Omega_{i}\right) .
$$

For the stress tensor we consider the finite element spaces $\mathcal{E}_{i, h}$ with dimension $N_{i}^{s}$ to approximate each subdomain component $\boldsymbol{\tau}_{i}$. These spaces are defined using elements of the type $\mathbb{P}^{1}$ that are continuous in $\bar{\Omega}_{i}$. The above spaces are defined by:

$$
\mathcal{E}_{i, h}=\left\{\boldsymbol{\sigma}_{i, h} \in \mathcal{E}_{i} \cap \mathbf{C}\left(\bar{\Omega}_{i}\right): \boldsymbol{\sigma}_{i, h_{\left.\right|_{K}}} \in \mathbb{P}^{1}, \forall K \in \mathcal{T}_{i, h}\right\}
$$

and

$$
\mathcal{E}_{i, h}^{0}=\left\{\boldsymbol{\sigma}_{i, h} \in \mathcal{E}_{i, h}: \boldsymbol{\sigma}_{i, h_{\mid \Gamma}}=\mathbf{0}\right\} .
$$

Let us also consider the basis functions

$$
\left\{\boldsymbol{\varphi}_{i, k}\right\}_{k=1, \ldots, N_{i}^{v}}, \quad\left\{\psi_{i, k}\right\}_{k=1, \ldots N_{i}^{p}} \quad \text { and } \quad\left\{\boldsymbol{\sigma}_{i, k}\right\}_{k=1, \ldots, N_{i}^{s}}
$$

for the finite element spaces associated to $\mathbf{V}_{h, i}, Q_{h, i}$ and $\mathcal{E}_{i, h}$, respectively.

Writing the variables $\left(\mathbf{u}_{i}, p_{i}\right)$ and $\boldsymbol{\tau}_{i}(i=1,2)$ and using the finite element bases of each space, we have

$$
\begin{aligned}
& \mathbf{u}_{i, h}= \sum_{k=1}^{N_{i}^{v}} U_{i, k} \boldsymbol{\varphi}_{i, k}=\sum_{k=1}^{N_{i, i n}^{v}} U_{i, k} \boldsymbol{\varphi}_{i, k}+\sum_{N_{i, i n}^{v}+1}^{N_{i}^{v}} U_{i, k} \boldsymbol{\varphi}_{i, k}, \\
& \mathbf{p}_{i, h}=\sum_{k=1}^{N_{i}^{p}} P_{i, k} \psi_{i, k}=\sum_{k=1}^{N_{i, i n}^{p}} P_{i, k} \psi_{i, k}+\sum_{N_{i, i n}^{p}+1}^{N_{i}^{p}} P_{i, k} \psi_{i, k}
\end{aligned}
$$

and

$$
\boldsymbol{\tau}_{i, h}=\sum_{k=1}^{N_{i}^{s}} T_{i, k} \boldsymbol{\sigma}_{i, k}=\sum_{k=1}^{N_{i, i n}^{s}} T_{i, k} \boldsymbol{\sigma}_{i, k}+\sum_{N_{i, i n}^{s}+1}^{N_{i}^{s}} T_{i, k} \boldsymbol{\sigma}_{i, k} .
$$

The last term that appears in all three summations refers to the variables defined on the interface. Let us denote by $N_{\Gamma}^{v}, N_{\Gamma}^{p}$ and $N_{\Gamma}^{s}$ the number of nodes on the interface for the velocity, pressure and stress tensor, respectively. We rewrite

$$
N_{i}^{v}=N_{i, i n}^{v}+N_{\Gamma}^{v}, \quad N_{i}^{p}=N_{i, i n}^{p}+N_{\Gamma}^{p} \quad \text { and } \quad N_{i}^{s}=N_{i, i n}^{s}+N_{\Gamma}^{s}
$$


where $N_{i, \text { in }}$ denotes the number of nodes in $\bar{\Omega}_{i} \backslash \Gamma$, for each variable. Using this separation of nodes that are in $\bar{\Omega}_{i} \backslash \Gamma$ or on $\Gamma$, we rewrite the previous basis functions, as follows, first for the velocity field

$$
\left\{\boldsymbol{\varphi}_{i, k}\right\}_{k=1, \ldots, N_{i, i n}^{v}} \quad \text { and } \quad\left\{\boldsymbol{\varphi}_{\Gamma, k}\right\}_{k=1, \ldots, N_{\Gamma}^{v}},
$$

then for the pressure

$$
\left\{\psi_{i, k}\right\}_{k=1, \ldots, N_{i, i n}^{p}} \quad \text { and } \quad\left\{\psi_{\Gamma, k}\right\}_{k=1, \ldots, N_{\Gamma}^{p}}
$$

and finally for the stress tensor

$$
\left\{\boldsymbol{\sigma}_{i, k}\right\}_{k=1, \ldots, N_{i, i n}^{v}} \quad \text { and } \quad\left\{\boldsymbol{\sigma}_{\Gamma, k}\right\}_{k=1, \ldots, N_{\Gamma}^{v}} .
$$

Considering the global-fixed point scheme (6) with a explicit linearization of the convective term and the discrete formulation of the Navier-Stokes equations in the multi-domain context, substituting the variables $\left(\mathbf{u}_{i}, p_{i}\right)(i=1,2)$ leads to the following system

$$
\left\{\begin{array}{l}
\mathbf{A}_{i} \mathbf{X}_{\mathbf{i}}=\mathbf{H}_{i}\left(\mathbf{U}_{i}, \mathbf{T}_{i}\right) \\
+ \text { t. c. on } \Gamma
\end{array}\right.
$$

and for the transport equation we have

$$
\left\{\begin{array}{l}
\mathbf{S}_{i}\left(\mathbf{U}_{i}\right) \mathbf{T}_{\mathbf{i}}=\mathbf{G}_{i}\left(\mathbf{U}_{i}, \mathbf{T}_{i}\right) \\
+ \text { t. c. on } \Gamma
\end{array}\right.
$$

where the previous matrices and vectors are defined in each subdomain $\Omega_{i}, i=1,2$ and $\mathbf{X}_{i}=\left(\mathbf{U}_{i}, \mathbf{P}_{i}\right)$.

3.3. Numerical scheme. The domain decomposition methods for the Navier-Stokes system and the transport equation are based on a Schwarz type algorithm [11]. This algorithm, Modified Schwarz Multiplicative Algorithm, consists in introducing two interface matrices that optimize the transmission conditions on $\Gamma$. We present here the procedure for the Navier-Stokes system.

In order to construct the two interface matrices we consider a reordering on the numeration of the nodes in each subdomain $\Omega_{i}$, which allow to rewrite the matrices $\mathbf{A}_{i}$ and the vector $\mathbf{H}_{i}$ as follows

$$
\mathbf{A}_{i}=\left(\begin{array}{cc}
\mathbf{A}_{i i} & \mathbf{A}_{i \Gamma} \\
\mathbf{A}_{\Gamma i} & \mathbf{A}_{\Gamma \Gamma}^{(i)}
\end{array}\right) \quad \text { and } \quad \mathbf{H}_{i}=\left(\begin{array}{c}
\mathbf{H}_{i, i n} \\
\mathbf{H}_{i, \Gamma}
\end{array}\right) \quad(i=1,2) .
$$

Let $\left\{\mathbf{e}_{k}\right\}_{k=1, \ldots, 2 N_{\Gamma}^{v}+N_{\Gamma}^{p}}$ be the corresponding canonical basis, and for $i=1$, 2 , let $\left(\mathbf{W}_{i, i n}, \mathbf{W}_{i, \Gamma}\right)$ be the solution of the following problem:

$$
\begin{cases}\mathbf{A}_{i i} \mathbf{W}_{i, \text { in }}+\mathbf{A}_{i \Gamma} \mathbf{W}_{i, \Gamma}=0 & \text { in } \Omega_{i} \\ \mathbf{W}_{i, \Gamma}=\mathbf{e}_{k} & \text { on } \Gamma\end{cases}
$$

The interface matrices $\mathcal{M}_{1}$ and $\mathcal{M}_{2}$ (corresponding to the Navier-Stokes system) are obtained through the following identities:

$$
\begin{aligned}
& \mathcal{M}_{1} \mathbf{e}_{k}=-\mathbf{A}_{\Gamma 2} \mathbf{W}_{2, \text { in }}-\mathbf{A}_{\Gamma \Gamma}^{2} \mathbf{W}_{2, \Gamma}, \\
& \mathcal{M}_{2} \mathbf{e}_{k}=-\mathbf{A}_{\Gamma 1} \mathbf{W}_{1, \text { in }}-\mathbf{A}_{\Gamma \Gamma}^{1} \mathbf{W}_{1, \Gamma} .
\end{aligned}
$$


Similarly, we construct two interface matrices $\mathcal{N}_{1}$ and $\mathcal{N}_{2}$, corresponding to the transport equation.

More precisely, at each step of the fixed-point algorithm stated previously, we apply the modified Schwarz multiplicative scheme to solve systems (9) and (10).

\section{Modified Schwarz Multiplicative Algorithm:}

- For $m \geq 0$, solve

\section{The Navier-Stokes system}

For $k=0, K$ :

- Given $\mathbf{U}_{2}^{k}=\left(\mathbf{U}_{2, \text { in }}^{k}, \mathbf{U}_{2, \Gamma}^{k}\right)$ and $\mathbf{T}_{1}^{m}$,

$$
\left\{\begin{array}{l}
\text { Find } \mathbf{U}_{1}^{k+1}=\left(\mathbf{U}_{1, \text { in }}^{k+1}, \mathbf{U}_{1, \Gamma}^{k+1}\right) \text { such that } \\
\mathbf{A}_{11} \mathbf{U}_{1, \text { in }}^{k+1}+\mathbf{A}_{1 \Gamma} \mathbf{U}_{1, \Gamma}^{k+1}=\mathbf{H}_{1, \text { in }}, \\
\mathbf{A}_{\Gamma 1} \mathbf{U}_{1, \text { in }}^{k+1}+\left(\mathbf{A}_{\Gamma \Gamma}^{1}-\mathcal{M}_{1}\right) \mathbf{U}_{1, \Gamma}^{k+1} \\
=\mathbf{H}_{\Gamma}-\mathbf{A}_{\Gamma 2} \mathbf{U}_{2, \text { in }}^{k}-\left(\mathbf{A}_{\Gamma \Gamma}^{2}+\mathcal{M}_{1}\right) \mathbf{U}_{2, \Gamma}^{k}
\end{array}\right.
$$

- Given $\mathbf{U}_{1}^{k+1}=\left(\mathbf{U}_{1, \text { in }}^{k+1}, \mathbf{U}_{1, \Gamma}^{k+1}, \mathbf{T}_{2}^{m}\right)$,

$$
\left\{\begin{array}{l}
\text { Find } \mathbf{U}_{2}^{k+1}=\left(\mathbf{U}_{2, \text { in }}^{k+1}, \mathbf{U}_{2, \Gamma}^{k+1}\right) \text { such that } \\
\mathbf{A}_{22} \mathbf{U}_{2, \text { in }}^{k+1}+\mathbf{A}_{2 \Gamma} \mathbf{U}_{2, \Gamma}^{k+1}=\mathbf{H}_{2, \text { in }} \\
\mathbf{A}_{\Gamma 2} \mathbf{U}_{2, \text { in }}^{k+1}+\left(\mathbf{A}_{\Gamma \Gamma}^{2}-\mathcal{M}_{2}\right) \mathbf{U}_{2, \Gamma}^{k+1} \\
=\mathbf{H}_{\Gamma}-\mathbf{A}_{\Gamma 1} \mathbf{U}_{1, \mathrm{in}}^{k+1}-\left(\mathbf{A}_{\Gamma \Gamma}^{1}+\mathcal{M}_{2}\right) \mathbf{U}_{1, \Gamma}^{k+1}
\end{array}\right.
$$

- Set $\mathbf{U}_{1}^{m}=\mathbf{U}_{1}^{k+1}$ and $\mathbf{U}_{2}^{m}=\mathbf{U}_{2}^{k+1}$.

\section{The transport equation}

For $k=0, K$ :

- Given $\mathbf{U}_{1}^{m}$ and $\left(\mathbf{T}_{2, \text { in }}^{k}, \mathbf{T}_{2, \Gamma}^{k}\right)$,

$$
\left\{\begin{array}{l}
\text { Find } \mathbf{T}_{1}^{k+1} \text { such that } \\
\mathbf{S}_{11} \mathbf{T}_{1, \text { in }}^{k+1}+\mathbf{S}_{1 \Gamma} \mathbf{T}_{1 \Gamma}^{k+1}=\mathbf{G}_{1, \text { in }}, \\
\mathbf{S}_{\Gamma 1} \mathbf{T}_{1, \text { in }}^{k+1}+\left(\mathbf{S}_{\Gamma \Gamma}^{1}-\mathcal{N}_{1}\right) \mathbf{T}_{1 \Gamma}^{k+1} \\
=\mathbf{G}_{\Gamma}-\mathbf{S}_{\Gamma 2} \mathbf{T}_{2, \text { in }}^{k}-\left(\mathbf{A}_{\Gamma \Gamma}^{2}+\mathcal{N}_{1}\right) \mathbf{T}_{2, \Gamma}^{k}
\end{array}\right.
$$


- Given $\mathbf{U}_{2}^{m}$ and $\left(\mathbf{T}_{1, \text { in }}^{k}, \mathbf{T}_{1, \Gamma}^{k}\right)$,

$$
\left\{\begin{array}{l}
\text { Find } \mathbf{T}_{2}^{k+1} \text { such that } \\
\mathbf{S}_{22} \mathbf{T}_{2, \text { in }}^{k+1}+\mathbf{S}_{2 \Gamma} \mathbf{T}_{2 \Gamma}^{k+1}=\mathbf{G}_{2, \text { in }} \\
\mathbf{S}_{\Gamma 2} \mathbf{T}_{2, \text { in }}^{k+1}+\left(\mathbf{S}_{\Gamma \Gamma}^{2}-\mathcal{N}_{2}\right) \mathbf{T}_{2 \Gamma}^{k+1} \\
=\mathbf{G}_{\Gamma}-\mathbf{S}_{\Gamma 1} \mathbf{T}_{1, \text { in }}^{k+1}-\left(\mathbf{S}_{\Gamma \Gamma}^{1}+\mathcal{N}_{2}\right) \mathbf{T}_{1, \Gamma}^{k+1}
\end{array}\right.
$$

- Set $\mathbf{T}_{1}^{m}=\mathbf{T}_{1}^{k+1}$ and $\mathbf{T}_{2}^{m}=\mathbf{T}_{2}^{k+1}$.

3. Set $\mathbf{U}_{i}^{0}=\mathbf{U}_{i}^{m}, \mathbf{T}_{i}^{0}=\mathbf{T}_{i}^{m}(i=1,2)$.

4. $m=m+1$.

To enhance the speed of convergence we next introduce in each subdomain a global block preconditioner.

4. Block preconditioners. Let us first consider a general framework for the block preconditioning of the Navier-Stokes system (see e.g. [9], [10], [5])

$$
\left(\begin{array}{ll}
\mathbf{F} & \mathbf{B} \\
\mathbf{B}^{T} & -\mathbf{C}
\end{array}\right) \mathbf{X}=\mathbf{H} .
$$

Let $\mathbf{P}_{R}$ be a right global preconditioner of the form

$$
\mathbf{P}_{R}=\left(\begin{array}{cc}
\mathbf{P}_{\mathbf{F}} & \mathbf{B} \\
0 & -\mathbf{P}_{\mathbf{S}}
\end{array}\right),
$$

where $\mathbf{P}_{\mathbf{F}}$ and $\mathbf{P}_{\mathbf{S}}$ are preconditioners for $\mathbf{F}$ and $\mathbf{S}=\mathbf{B}^{T} \mathbf{F}^{-1} \mathbf{B}+\mathbf{C}$ (the corresponding Schur complement), respectively. Since

$$
\mathbf{P}_{R}^{-1}=\left(\begin{array}{cc}
\mathbf{P}_{\mathbf{F}}^{-1} & \mathbf{P}_{\mathbf{F}}^{-1} \mathbf{B} \mathbf{P}_{\mathbf{S}}^{-1} \\
0 & -\mathbf{P}_{\mathbf{S}}^{-1}
\end{array}\right),
$$

then the linear system (21) is equivalent to

$$
\left\{\begin{array}{cc}
\left(\begin{array}{cc}
\mathbf{F} \mathbf{P}_{\mathbf{F}}^{-1} & {\left[\mathbf{F P}_{\mathbf{F}}^{-1}-\mathbf{I}_{d}\right) \mathbf{B} \mathbf{P}_{\mathbf{S}}^{-1}} \\
\mathbf{B}^{T} \mathbf{P}_{\mathbf{F}}^{-1} & \mathbf{S P}_{\mathbf{S}}^{-1}
\end{array}\right) \mathbf{Y}=\mathbf{H}, \\
\mathbf{P}_{R} \mathbf{X}=\mathbf{Y} .
\end{array}\right.
$$

In a first step, this approach is used to solve system (16). Taking into account the fact that $\mathbf{A}_{i i}(i=1,2)$ has the same structure as $\mathbf{A}$, i.e.

$$
\mathbf{A}_{i i}=\left(\begin{array}{cc}
\mathbf{A}_{i i}^{*} & \mathbf{B}_{i i}^{*} \\
\left(\mathbf{B}_{i i}^{*}\right)^{T} & \mathbf{0}
\end{array}\right),
$$


and choosing $\mathbf{F}=\mathbf{P}_{\mathbf{F}}=\mathbf{A}_{i i}^{*}, \mathbf{S}=\mathbf{P}_{\mathbf{S}}=\left(\mathbf{B}_{i i}^{*}\right)^{T}\left(\mathbf{A}_{i i}^{*}\right)^{-1} \mathbf{B}_{i i}^{*}$, we can easily see that system (16) is equivalent to

$$
\left\{\begin{array}{l}
\left(\begin{array}{cc}
\mathbf{I} & 0 \\
\left(\mathbf{B}_{i i}^{*}\right)^{T}\left(\mathbf{A}_{i i}^{*}\right)^{-1} & \mathbf{I}
\end{array}\right) \mathbf{Y}_{i n}=-\mathbf{A}_{i \Gamma} \mathbf{e}_{k} \\
\left(\begin{array}{cc}
\mathbf{A}_{i i}^{*} & \mathbf{B}_{i i}^{*} \\
\mathbf{0} & -\mathbf{S}
\end{array}\right) \mathbf{W}_{i, i n}=\mathbf{Y}_{i n}
\end{array}\right.
$$

The new system is solved using a GMRES method [20]. In this case it is known that the first system converges in two or three iterations and that the convergence is independent of the mesh [13].

In the same spirit, this preconditioning approach is applied to the matrices

$$
\mathbf{A}_{i}=\left(\begin{array}{ll}
\mathbf{A}_{i i} & \mathbf{A}_{i \Gamma} \\
\mathbf{A}_{\Gamma i} & \mathbf{A}_{\Gamma \Gamma}^{(i)}-\mathcal{M}_{i}
\end{array}\right)
$$

associated to the Navier-Stokes systems in the subdomains $\Omega_{i}(i=1,2)$. The corresponding systems can be formally written as

$$
\mathbf{A}_{i} \mathbf{U}_{i}=\mathbf{H}_{i} \quad(i=1,2) .
$$

Let $\mathbf{P}_{A_{i}}$ be the right preconditioner for $\mathbf{A}_{i}$ defined by

$$
\mathbf{P}_{A_{i}}=\left(\begin{array}{cc}
\mathbf{P}_{A_{i i}} & \mathbf{A}_{i \Gamma} \\
\mathbf{0} & -\mathbf{P}_{S_{i, \Gamma}}
\end{array}\right)
$$

where $\mathbf{P}_{A_{i i}}$ and $\mathbf{P}_{S_{i, \Gamma}}$ are preconditioners for $\mathbf{A}_{i i}$ and $\mathbf{S}_{i, \Gamma}=\mathbf{A}_{\Gamma i}\left(\mathbf{A}_{i i}\right)^{-1} \mathbf{A}_{i \Gamma}-\mathbf{A}_{\Gamma \Gamma}^{(i)}+\mathcal{M}_{i}$. Problem (23) is equivalent to

$$
\left\{\begin{array}{l}
\mathbf{A}_{i} \mathbf{P}_{A_{i}}^{-1} \mathbf{Y}_{i}=\mathbf{H}_{i} \\
\mathbf{P}_{A_{i}} \mathbf{U}_{i}=\mathbf{Y}_{i}
\end{array}\right.
$$

where

$$
\mathbf{A}_{i} \boldsymbol{P}_{A_{i}}^{-1}=\left(\begin{array}{cc}
\mathbf{A}_{i i} \mathbf{P}_{A_{i i}}^{-1} & \left(\mathbf{A}_{i i} \mathbf{P}_{A_{i i}}^{-1}-\mathbf{I}_{d}\right) \mathbf{A}_{i \Gamma} \mathbf{P}_{S_{i, \Gamma}}^{-1} \\
\mathbf{A}_{\Gamma i} \mathbf{P}_{A_{i i}}^{-1} & S_{i, \Gamma} \mathbf{P}_{S_{i, \Gamma}}^{-1}
\end{array}\right)
$$

5. Numerical results. To validate our numerical code we consider the flow of an Oldroyd-B fluid in a planar contraction 4:1 (or abrupt-contraction) geometry. This test problem has been studied by many authors, since 1988, and recognized as a good benchmark for validation of numerical codes to simulate viscoelastic flows, see e.g. Saramito 
[22], Oliveira and Pinho [16] and Alves et al. [1]. The problem is defined as follows

$$
\begin{cases}\text { Find }(\mathbf{u}, p, \boldsymbol{\tau}) \text { such that } & \\ -(1-\varepsilon) \Delta \mathbf{u}+\nabla p+\mathcal{R} e(\mathbf{u} \cdot \nabla) \mathbf{u}=\nabla \cdot \boldsymbol{\tau} & \text { in } \Omega \\ \nabla \cdot \mathbf{u}=0 & \text { in } \Omega \\ \boldsymbol{\tau}+\mathcal{W} e \mathbf{u} \cdot \nabla \boldsymbol{\tau}-\mathcal{W} e\left(\boldsymbol{\tau} \nabla \mathbf{u}+(\nabla \mathbf{u})^{T} \boldsymbol{\tau}\right)=2 \epsilon D(\mathbf{u}) & \text { in } \Omega,\end{cases}
$$

where the domain $\Omega$ is defined in Figure 1.

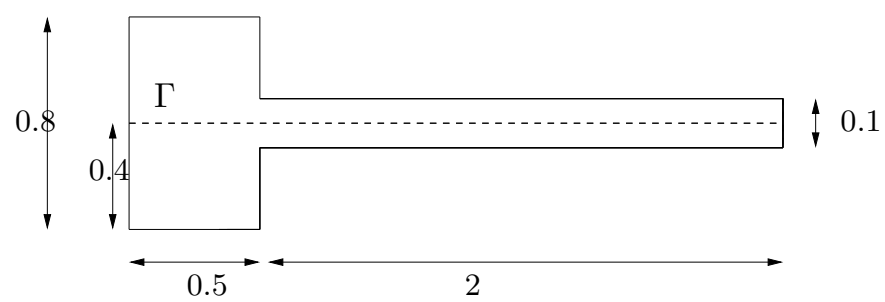

Fig. 1. Computational domain of a 4:1 - abrupt contraction

In order to close the system of PDE's we need to impose suitable boundary conditions for the velocity field and also for the stress tensor (due to memory effects). At the inflow boundary we consider a parabolic profile for the velocity field, and on the wall we consider no-slip conditions. We suppose that the downstream exit is long enough so that at the outlet a fully developed Poiseuille flow can be prescribed. The parabolic profiles that we consider were obtained in [22].

- Inflow conditions:

$$
\begin{gathered}
\mathbf{u}=\left(u^{1}, v^{1}\right)=0.0125\left(1-\left(\frac{y}{0.4}\right)^{2}, 0\right) \\
\tau^{11}=\frac{25}{1024} \epsilon \mathcal{W} e y^{2} \\
\tau^{12}=-\frac{5}{32} \epsilon \mathcal{W} e y^{2} \\
\tau^{22}=0
\end{gathered}
$$

- Outflow conditions:

$$
\mathbf{u}=\left(u^{1}, v^{1}\right)=0.05\left(1-\left(\frac{y}{0.1}\right)^{2}, 0\right) .
$$

- Conditions on the wall:

$$
\mathbf{u}=\left(u^{1}, v^{1}\right)=(0,0) .
$$

The numerical tests were performed with PDDM (Preconditioned Domain Decomposition Method) for $\mathcal{R} e=1$ and $\mathcal{W} e=0.1$ and considering a decomposition of the domain $\Omega$ into 
two subdomains, $\Omega_{1}$ and $\Omega_{2}$ (cf. Figure 1). In each subdomain we have considered a mesh with 808 elements. Observing the results of figures 2 and 3, we can see that there exists a discontinuity on the interface for each variable, which vanishes with the convergence of our method.

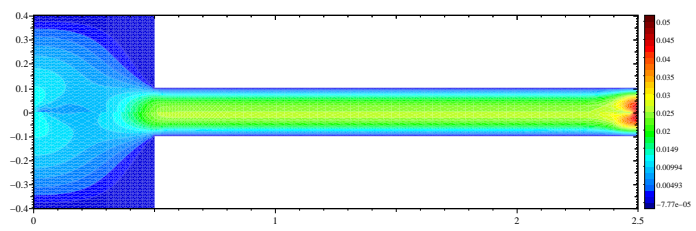

(a) first iteration

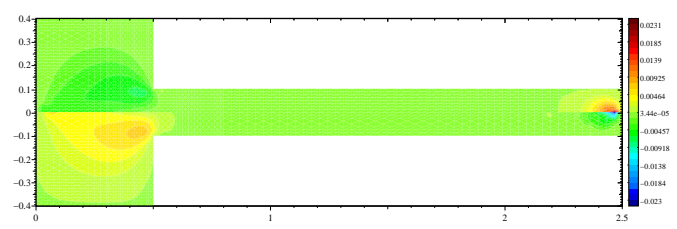

(c) first iteration

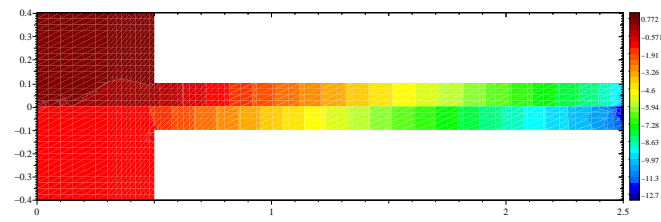

(e) first iteration

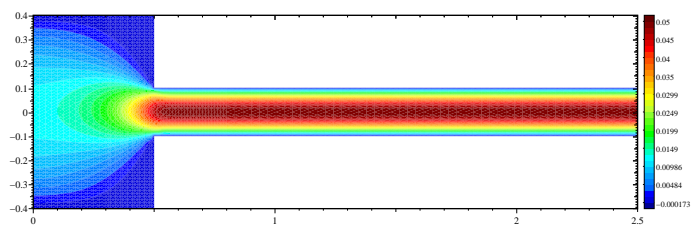

(b) last iteration

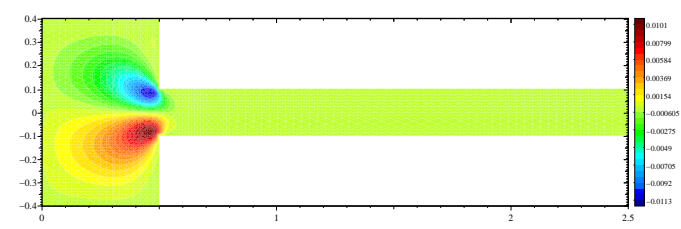

(d) last iteration

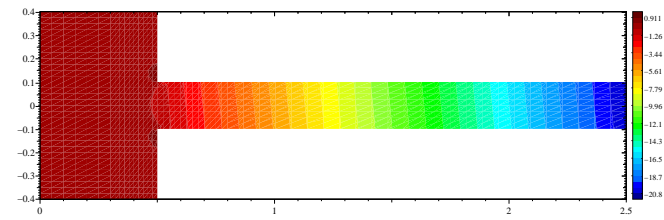

(f) last iteration

Fig. 2. Contour plots for $\mathcal{R} e=1$ and $\mathcal{W} e=0.1$ using PDDM: first component of the velocity field (a) and (b); second component of the velocity field (c) and (d); pressure field (e) and (f).

This benchmark has two critical zones, the two reentrant corners, which are one of the reasons for convergence failure when high values of the Weissenberg parameter are considered. With our tests we could observe that due to the dimensions of our domain and to the inlet condition, the problem is very sensitive to changes in the Weissenberg number. This behaviour can be checked in Figure 4, where the derivatives of tensor $\tau^{11}$ with respect to $x$ grow with the variation of the Weissenberg number.

Analyzing Figure 5 we can observe, as expected, two vortices in both corners of the abrupt contraction. 


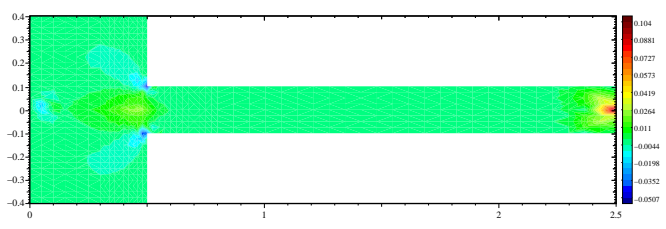

(a) first iteration

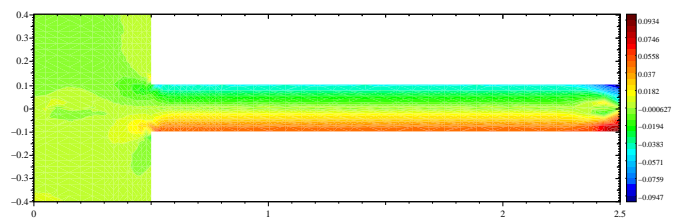

(c) first iteration

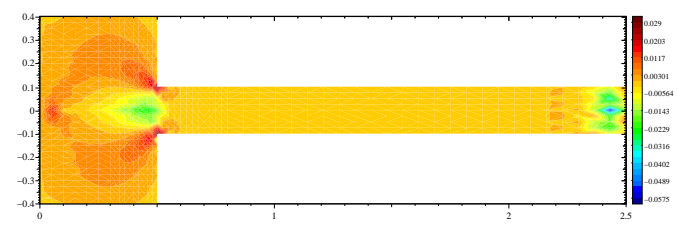

(e) first iteration

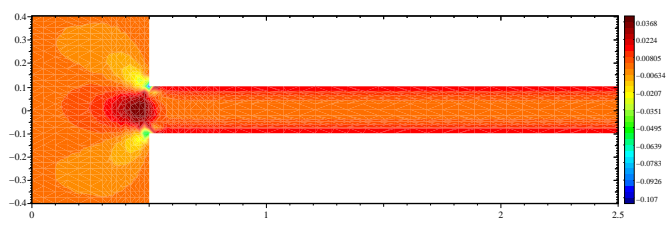

(b) last iteration

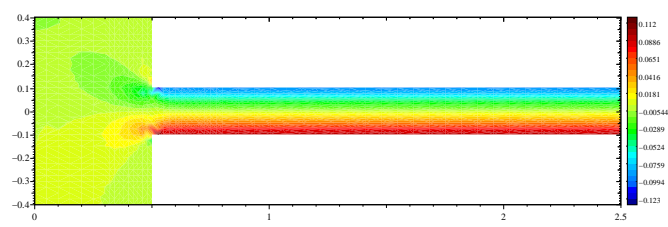

(d) last iteration

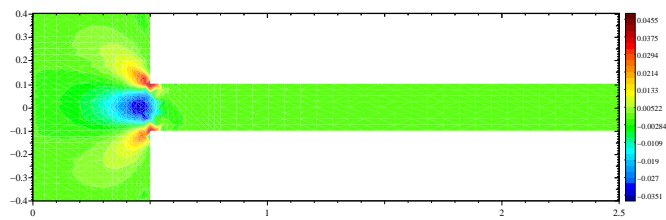

(f) last iteration

Fig. 3. Contour plots of the stress tensor components, for $\mathcal{R} e=1$ and $\mathcal{W} e=0.1$ using PDDM: component $\tau^{11}(\mathrm{a})$ and (b); component $\tau^{12}=\tau^{21}(\mathrm{c})$ and (d): component $\tau^{22}$ (e) and (f).

6. Conclusions. In this paper we use Domain Decomposition Methods (DDM) to solve the equations of motion of incompressible non-Newtonian viscoelastic Oldroyd-B fluids. Based on the decomposition of the original problem into a Navier-Stokes system and a tensorial transport equation, the method has been applied to both systems, considered as auxiliar problems. The Navier-Stokes equations were solved using several types of block preconditioners and the corresponding results indicate a significant reduction in CPU time when compared to those obtained using a global approximation of the problem. The numerical techniques developed for both the Navier-Stokes system and the transport equation have been coupled to solve the Oldroyd-B model. The validation of our numerical scheme was done by considering a benchmark test for flow in a 4:1 abrupt contraction. This is known as a good benchmark to study viscoelastic flows, due in particular to the existence of vortices in the two reentrant corners, which are very sensitive to variations in the Weissenberg number. The extension of these numerical techniques to 


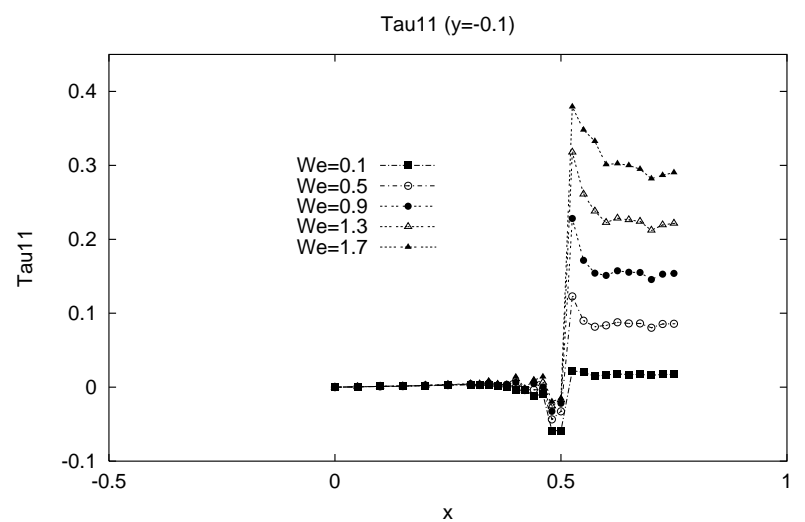

Fig. 4. Values of the stress component $\tau^{11}$ for $\mathcal{R} e=1, \mathcal{W} e=0.1,0.5,0.9,1.3,1.7$, along the line $y=-0.1$ with $x \in[0,0.7]$.
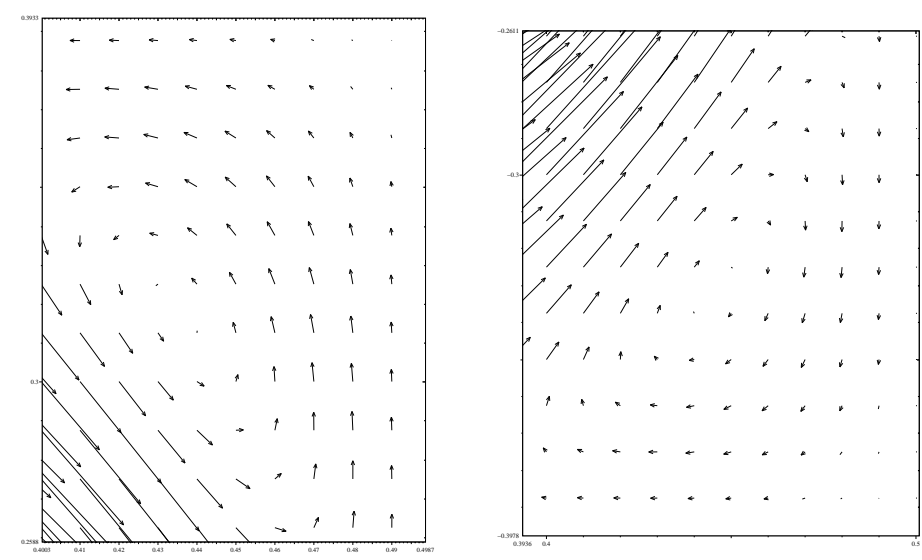

Fig. 5. Zoom of the vector field plot for abrupt contraction, upper corner (left) lower corner (right), for $\mathcal{R} e=1, \mathcal{W} e=1$ and $\epsilon=0.1$.

the decomposition of the domain into more than two subdomains in view of the parallel computing with several processors, will be the subject of a forthcoming paper.

\section{References}

[1] M. A. Alves, P. J. Oliveira and F. T. Pinho, Benchmark solutions for the flow of Oldroyd-B and PTT fluids in planar contractions, J. Non-Newtonian Fluid Mech. 110 (2003), 45-75.

[2] N. Arada, L. Borges and A. Sequeira, A preconditioned domain decomposition method for the simulation of viscoelastic flows, WSEAS-Transactions on Mathematics 5 (2006), 289-296. 
[3] M. Bensaada, D. Esselaoui and D. Sandri, Stabilization method for continuous approximation of transient convection problem, Numerical Methods for Partial Differential Equations 21 (2004), 170-189.

[4] L. Borges, Domain Decomposition Methods for Non-Newtonian Fluids, Phd Thesis, Universidade Técnica de Lisboa, Instituto Superior Técnico, 2006.

[5] H. Elman, D. Silvester and A. Wathen, Block preconditioners for the discrete incompressible Navier-Stokes equations, Int. J. Numer. Methods Fluids 40 (2002), 333-344.

[6] V. Ervin and W. Miles, Approximation of time-dependent viscoelastic fluid flow: SUPG approximations, SIAM, J. Numer. Anal. 41 (2003), 457-486.

[7] V. Girault and P. A. Raviart, Finite Element Methods for Navier-Stokes Equations, Computational Mathematics, Springer-Verlag, Berlin, 1986.

[8] R. Glowinsky and F. Wheeler, Domain decomposition and mixed element methods for elliptic problems, in: Proc. First International Symposium on Domain Decomposition Methods for Partial Differential Equations, SIAM, Philadelphia, 1988.

[9] D. Loghin and A. Wathen, Schur complement for the Navier-Stokes equations, Int. J. Numer. Methods Fluids 40 (2002), 403-412.

[10] D. Kay, D. Loghin and A. Wathen, A preconditioner for the steady-state Navier-Stokes equations, SIAM J. Sci. Comput. 24 (2002), 237-256.

[11] C. Japhet, Méthode de décomposition de domaine et conditions aux limites artificielles en mécanique des fluides: Méthode optimisée d'ordre 2, Phd Thesis, Université Paris 13, 1998.

[12] C. Johnson and J. Pitkaranta, An analysis of the discontinuous Galerkin method for a scalar hyperbolic equation, Math. Comp. 46 (1986), 1-26.

[13] M. Murphy, G. Golub and A. Wathen, A note on preconditioning for indefinite linear systems, SIAM J. Sci. Comput. 21 (2000), 1969-1972.

[14] P. Lesaint and P. A. Raviart, On a finite element method for solving the neutron transport equation, in: C. de Boor (ed.), Mathematical Aspects of Finite Elements in Partial Differential Equations, New York, Academic Press, 1974, 89-123.

[15] K. Najib and D. Sandri, On a decoupled algorithm for solving a finite element problem for the approximation of viscoelastic fluid flow, Numerische Mathematik 72 (1995), 223-238.

[16] P. Oliveira and F. Pinho, Numerical procedure for the computation of fluid flow with arbitrary stess-strain relationships, Numer. Heat Transfer B 35 (1999), 295-319.

[17] R. G. Owens and T. N. Phillips, Computational Rheology, Imperial College Press/World Scientific, London, UK, 2002.

[18] A. Quarteroni and A. Valli, Domain Decomposition Methods for Partial Differential Equations, Oxford University Press, Oxford, 1999.

[19] M. Renardy, Mathematical Analysis of Viscoelastic Flows, CBMS 73, SIAM, Philadelphia, 2000 .

[20] Y. Saad and M. H. Schultz, A generalized minimal residual algorithm for solving nonsymmetric linear systems, SIAM J. Sci. Stat. Comput. 7 (1986), 859-869.

[21] D. Sandri, On a FEM method for a linearized version of the Oldroyd's problem, Comput. Meth. Appl. Mech. Engrg. 191 (2002), 5054-5065.

[22] P. Saramito, A new $\theta$-scheme algorithm and incompressible FEM for vicoelastic fluids flows, Mathematical Modelling and Numerical Analysis 48 (1994), 1-34.

[23] P. Le Tallec, Y. De Roeck and M. Vidrascu, Domain decomposition methods for large linearly elliptic three-dimensional problems. J. Comput. Appl. Math. 34 (1991), 93-117. 